\title{
Cobalt plaque versus enucleation for uveal melanoma: comparison of survival rates
}

\author{
KIMBERLEY S ADAMS,' DAVID H ABRAMSON ${ }^{2}$ ROBERT M ELLSWORTH, \\ BARRETT G HAIK, ${ }^{2}$ MICHAEL BEDFORD, ${ }^{3}$ SAMUEL PACKER ${ }^{4}$ \\ JOHANNA SEDDON, ${ }^{5}$ DANIEL ALBERT, ${ }^{5}$ AND LELA POLIVOGIANIS
}

From the Departments of Ophthalmology, 'Manhattan Eye, Ear and Throat Hospital; the ${ }^{2}$ New York Hospital-® Cornell Medical Center, New York, New York; ${ }^{3}$ St Bartholomew's Hospital, London, England; ${ }^{4}$ North Shore $\rightarrow$ Hospital, Manhasset, New York; and ${ }^{5}$ Harvard Medical School, Boston, Massachusetts, USA

SUMmaRY Two hundred and twenty-three patients treated by cobalt plaque for uveal melanoma $\frac{\overparen{\circ}}{\circ}$ were compared with 416 patients treated by enucleation for uveal melanoma in terms of patient survival. The median follow-up time for the patients treated by cobalt plaque was 4.3 years. $v$ Kaplan-Meier survival curves were calculated up to five years following treatment based on time toco tumour-related deaths. Cox's proportional hazards multivariate analysis was performed to ${ }_{\circ}^{+}$ determine which variables were related to melanoma-related deaths while controlling for age, size,, and location of the tumours. Statistically significant predictive factors were location of tumour ande largest tumour dimension. There was not a statistically significant difference in survival between $\frac{\varsigma}{<}$ patients treated by cobalt plaque and those treated by enucleation.

Many studies have explored prognostic factors and survival rates for patients with malignant melanoma of the choroid and ciliary body treated by enucleation. ${ }^{1-4} \mathrm{~A}$ few studies have investigated similar factors with respect to treatment with cobalt-60 plaque therapy, but these have involved small numbers of patients and short follow-up times. ${ }^{\text {s-9 }}$ There has been, and continues to be, much discussion concerning the pros and cons of various types of treatment for malignant melanoma. The most basic issue in these debates would appear to be patient survival, yet no long-term study on a large patient population comparing treatment by enucleation with cobalt plaque, in terms of survival, exists. Comparison of prior studies is difficult: tumour dimensions have been variable, different methods of analysis have been used, patient numbers are small, and follow-up time short. ${ }^{1-9}$ This study compares survival in terms of melanoma-related deaths between cobalt- 60 plaquetreated patients (with long-term follow-up from multiple institutions in the United States and one in England) and patients treated by enucleation from the Massachusetts Eye and Ear Infirmary.

Correspondence to Kimberley Adams, MD, Manhattan Eye, Ear and Throat Hospital, Department of Ophthalmology, 210 East 64th Street, New York, New York 10021, USA.

\section{Material and methods}

Three groups of patients with choroidal and ciliary body melanoma were compared for survival. Two $\vec{F}$ hundred and sixty-seven patients, with a diagnosis of 3 uveal melanoma from 1953 to 1973, treated by enucleation were identified from the records of theo Eye Pathology Laboratory at the Massachusetts Eye and Ear Infirmary (MEEI). Thirty-two patients witho histological extrascleral extension were excluded, $\frac{0}{3}$ leaving a total of 235 cases for group I. These subjectshave been described elsewhere ${ }^{4}$ and were part of a group of 267 cases with known survival status out of a total of 431 eligible patients.

Group II included 161 patients from MEEI with aD diagnosis of choroidal and ciliary body melanoma․ whose eyes were enucleated from 1975 to 1981 . From a total of 231 patients treated by enucleation during this period 20 were excluded because of extrascleralo extension, and the survival status was unknown forw another 50 patients.

Group III consisted of 223 patients with a diagnosise of uveal melanoma on the basis of clinical examina- $-\bar{\Phi}$ tion, indirect ophthalmoscopy, ultrasonography, and ${ }^{\text {f }}$ fluorescein angiography, treated by cobalt-60 plaque $\frac{T}{-}$ at several institutions: 142 cases from 1968 to $1984 \stackrel{\circ}{\vec{D}}$ identified from the records at the New York Hospita 
and Manhattan Eye, Ear and Throat Hospital; seven cases treated from 1975 to 1977 identified at North Shore University Hospital, New York; and 74 cases treated from 1954 to 1981 identified at St Bartholomew's Hospital, London. No patients had known extrascleral extension at the time of cobalt plaquing and all patients were included in the study.

Survival status, the date and cause of death, and metastatic disease status were determined up to 1 May 1983 in the enucleation groups and up to June 1984 in the cobalt plaque group by chart review, contact with patients, and their families, as well as contact with physicians. Survival status was recorded as alive without known metastases, alive with metastases, dead secondary to metastatic uveal melanoma, or dead from other causes. Metastases were confirmed by liver function abnormalities biopsy, or necropsy.

Tumour dimensions for the enucleation group were obtained from review of histopathological studies: the largest tumour diameter in millimetres in contact with the sclera, and elevation of the tumour in millimetres measured on the largest section available. For the cobalt plaque group the tumour dimensions were determined by visualisation by indirect ophthalmoscopy or by ultrasonographic measurements. The largest tumour dimension for each tumour, either diameter or height, was used in the analysis.

The location of the anterior margin of the tumour was coded as posterior to the equator or anterior to the equator, with or without involvement of the ciliary body.

Survival curves, based on deaths from uveal melanoma for the first five years after treatment for all three treatment groups, were calculated by the Kaplan-Meier technique. ${ }^{10}$ Cox's proportional hazards multivariate analysis" ${ }^{\prime \prime}$ was performed to determine which variables were independently associated with melanoma-related deaths. The four variables considered in the Cox model were: (1) treatment: cobalt-60 plaque, enucleation 1953-73, and enucleation $1975-81$; (2) age: less than 40 years, 40-59 years, 60 years and older; (3) location of tumour: posterior to the equator or anterior to the equator; (4) largest tumour dimension (either diameter or height): small (up to $11 \mathrm{~mm}$ ), medium (11-15 mm), or large (16 mm or greater).

The statistical analyses were performed through the computer facilities of the Department of Ophthalmology at the Massachusetts Eye and Ear Infirmary.

\section{Results}

The median age at enucleation of patients with uveal
Table 1 Characteristics of tumours and patients according to treatment group

\begin{tabular}{|c|c|c|c|c|c|c|}
\hline \multirow{2}{*}{$\begin{array}{l}\text { Variable } \\
\text { Age (yr) }\end{array}$} & \multicolumn{2}{|c|}{ Cobalt plaque } & \multicolumn{2}{|c|}{$\begin{array}{l}\text { Enucleation } \\
\text { group I } \\
(1953-73)\end{array}$} & \multicolumn{2}{|c|}{$\begin{array}{l}\text { Enucleation } \\
\text { group II } \\
(1975-81)\end{array}$} \\
\hline & $n$ & $\%$ & $n$ & $\%$ & $n$ & $\%$ \\
\hline$<40$ & 28 & $12 \cdot 6$ & 18 & $7 \cdot 7$ & 17 & $10 \cdot 6$ \\
\hline $40-49$ & 35 & $15 \cdot 7$ & 49 & $20 \cdot 8$ & 16 & 9.9 \\
\hline $50-59$ & 61 & $27 \cdot 4$ & 62 & $26 \cdot 4$ & 34 & $21 \cdot 1$ \\
\hline $60-69$ & 51 & $22 \cdot 9$ & 69 & $29 \cdot 4$ & 49 & $30 \cdot 4$ \\
\hline $70+$ & 46 & $20 \cdot 6$ & 36 & $15 \cdot 3$ & 32 & 19.9 \\
\hline Unknown & 2 & 0.9 & 1 & 0.4 & 13 & $8 \cdot 1$ \\
\hline \multicolumn{7}{|l|}{ Location } \\
\hline Anterior & 67 & $30 \cdot 0$ & 78 & $33 \cdot 2$ & 79 & $49 \cdot 1$ \\
\hline Posterior & 154 & $69 \cdot 1$ & 138 & $58 \cdot 7$ & 80 & $49 \cdot 7$ \\
\hline Unknown & 2 & 0.9 & 19 & $8 \cdot 1$ & 2 & $1 \cdot 2$ \\
\hline \multicolumn{7}{|c|}{ Largest dimension $(\mathrm{mm})$} \\
\hline$<11$ & 152 & $68 \cdot 2$ & 113 & $48 \cdot 1$ & 68 & $42 \cdot 2$ \\
\hline $11-15$ & 67 & $30 \cdot 0$ & 87 & $37 \cdot 0$ & 71 & $44 \cdot 1$ \\
\hline $16+$ & 3 & $1 \cdot 3$ & 20 & $8 \cdot 5$ & 21 & $13 \cdot 0$ \\
\hline Unknown & 1 & 0.4 & 15 & $6 \cdot 4$ & 1 & $0 \cdot 6$ \\
\hline
\end{tabular}

melanoma in group I (1953-73) was $58 \cdot 5$ years with a range of 18 to 95 years; in group II (1975-81), the median was 60 years with a range of 10 to 94 years. The age at cobalt plaque treatment for choroidal and ciliary body melanoma patients in group III ranged from 22 years to 84 years, with a median of 57.0 years. For two of the 223 cobalt plaque patients age at the time of treatment was not available.

Table 1 shows the distribution of characteristics according to treatment group.

The follow-up time in enucleation group I ranged from 100 days to 28 years, with a median of 9.3 years. In group II the follow-up time ranged from 90 days to 8.5 years, with a median of 3.3 years. In group III, the cobalt plaque group, the follow-up time ranged from 30 days to 30 years, with a median of 4.3 years.

Thirty-six of the 223 patients treated by cobalt plaque for uveal melanoma underwent subsequent enucleation. Of these 36 patients 4 died of metastatic melanoma and one of other causes. Among the 188 patients treated only by cobalt plaque there were 18 deaths from metastatic disease and 11 from other causes. The analyses of patient survival comparing cobalt plaque treatment with enucleation were performed for all 223 cobalt plaque patients, including those 36 who underwent eventual enucleation. A further analysis was also made comparing the two enucleation groups, I and II, with the cobalt plaque group, excluding those with subsequent enucleation.

SURVIVAL CURVES

Kaplan-Meier survival curves were calculated from tumour deaths for the first five years after treatment for all three treatment groups (Fig. 1); for only small 


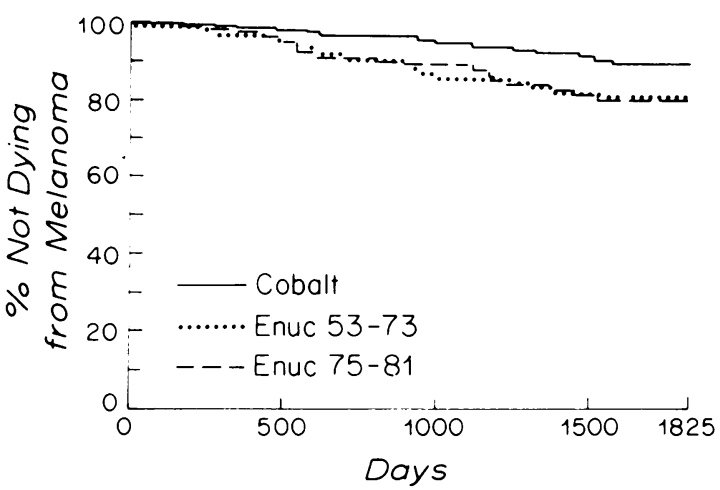

Fig. 1 Time to tumour death for all patients treated by cobalt plaque and enucleation.

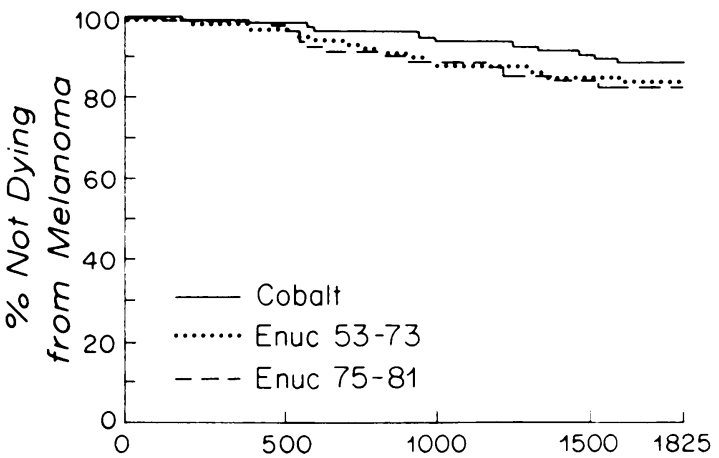

Fig. 2 Time to tumour death for all patients with small and medium tumours treated by cobalt plaque and enucleation.

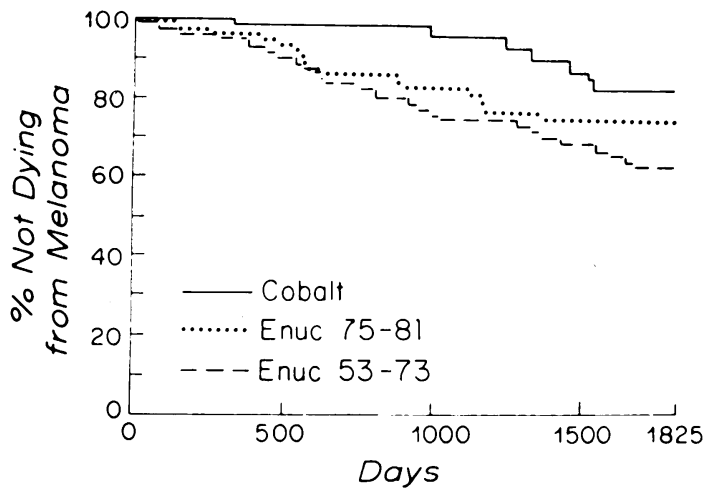

Fig. 3 Time to tumour death for all patients with anterior tumours treated by cobalt plaque and enucleation.

and medium tumours (Fig. 2); and for anteriorly located tumours (Fig. 3). Cobalt plaque treated patients showed significantly better survival than either enucleation group (Fig. 1), and there was a statistically significant difference in survival for anterior tumours between cobalt treated patients and patients treated by enucleation from 1953 to 1973 응 (Fig. 3). Other comparisons were not significantly $\underset{P}{?}$ different.

\section{VARIABLES RELATED TO SURVIVAL}

Cox's proportional hazards multivariate analysis, $\overrightarrow{\overline{\vec{D}}}$ including the variables previously mentioned (treat- $\overrightarrow{0}$ ment, age, location of tumour, largest tumour $\frac{c}{\sigma}$ dimension), showed that anterior location and $\overline{\bar{\omega}}$ largest tumour dimensions (11-15 $\mathrm{mm}$ and $16 \mathrm{~mm} \Phi$ and greater) were significant prognostic factors for melanoma related deaths (Table 2 ). Patients with $\infty$ anterior tumours were twice as likely to die from $\overrightarrow{0}$ melanoma in the first five years of follow-up as were $\overrightarrow{-}$ patients with posterior tumours. In addition patients with tumours with $11-15 \mathrm{~mm}$ as the largest tumour $\overline{0}$. dimension were 1.86 times as likely to die of tumour? as patients with tumours $1-10 \mathrm{~mm}$, and patients with $N$ tumours $16 \mathrm{~mm}$ or greater had a rate of melanoma $-v$ death 3.3 times that of patients with a tumour 1-10 $\overrightarrow{0}$ $\mathrm{mm}$. When these and other factors previously mentioned were controlled, there was no significant $\stackrel{5}{\longrightarrow}$ difference in survival between the cobalt plaque $\vec{c}$ treated patients and enucleation. Thirty-six of the 223 cobalt plaque treated patients eventually underwent enucleation. When these patients were $\stackrel{\infty}{\infty}$ excluded from the analyses, Kaplan-Meier curves showed no difference in survival between cobalt plaque patients and either enucleation group. This group was also analysed by means of the Cox model, which showed results similar to the analysis pre- ̊ sented above, including all cobalt plaque patients.

\section{Discussion}

Previous studies of patient survival in choroidal and ciliary body melanoma have largely been directed

Table 2 Results of Cox model analysis for deaths related to uveal melanoma

\begin{tabular}{|c|c|c|c|}
\hline Variable & Comparison & $\begin{array}{l}\text { Estimated } \\
\text { rate ratio* }\end{array}$ & $\begin{array}{l}95 \% \\
\text { Confidence } \\
\text { interval }\end{array}$ \\
\hline \multirow[t]{2}{*}{ Treatment } & $\begin{array}{l}\text { Enucleation group I vs } \\
\text { cobalt }\end{array}$ & $1 \cdot 07$ & $0.59-1.95$ \\
\hline & $\begin{array}{l}\text { Enucleation group } 2 \text { vs } \\
\text { cobalt }\end{array}$ & 0.77 & $0 \cdot 39-1 \cdot 51$ \\
\hline \multirow[t]{2}{*}{ Age } & $40-59$ vs $<40$ & $0 \cdot 84$ & $0 \cdot 34-2 \cdot 05$ \\
\hline & $60+v s<40$ & $1 \cdot 64$ & $0 \cdot 70-3 \cdot 87$ \\
\hline \multirow{2}{*}{$\begin{array}{l}\text { Location } \\
\text { Largest tumour } \\
\text { dimension }\end{array}$} & Anterior vs posterior & $2 \cdot 07$ & $1 \cdot 27-3 \cdot 37$ \\
\hline & $\begin{array}{l}11-15 \text { vs }<11 \\
16+v s<11\end{array}$ & $\begin{array}{l}1 \cdot 80 \\
3 \cdot 31\end{array}$ & $\begin{array}{l}1 \cdot 06-3 \cdot 04 \\
1 \cdot 67-6 \cdot 55\end{array}$ \\
\hline
\end{tabular}

*Estimated by the antilogarithm of the regression coefficients from proportional hazards model, with adjustment for all other variables presented. 
toward one type of treatment. In terms of enucleation Flocks et al. ' in 1955 had 73 out of 210 melanoma related deaths, McLean et al. ${ }^{2}$ in 1977 had 76 out of 217, Shammas and Blodi ${ }^{3}$ in 1977 had 87 out of 293, and Seddon and Albert ${ }^{4}$ in 1983 had 110 out of 274, with an average overall mortality of $34 \%$.

Studies of patient survival with cobalt plaque treatment include those of Stallards in 1966 with six melanoma related deaths out of 100, MacFaul ${ }^{6}$ in 1977 with 12 out of 107, Ellsworth' in 1978 with five out of 47, Migdal $^{8}$ in 1983 with five out of 90 , and Brady and Shields ${ }^{9}$ in 1984 with three out of 100 , with an average overall mortality of $7 \%$. Variable follow-up time makes meaningful comparison of these studies difficult.

A prior study comparing mortality data on a group of 100 patients treated by cobalt plaque with 100 patients treated by enucleation matched for age, sex, size of tumour, and follow-up from the HahnemannWills Eye Hospital Group indicated for the cobalt plaque group a death rate of $0 \%$ for small, $2 \cdot 4 \%$ for medium-sized, and $3.8 \%$ for large-sized melanomas. For their enucleation group death rates of $8 \cdot 3 \%$, $4.5 \%$, and $22 \%$ respectively were found. ${ }^{9}$

In contrast Gass $^{12}$ found a $50 \%$ probability of melanoma related death within five years after cobalt plaque in 21 patients, compared with $16 \%$ after enucleation in 27 patients.

Our study compares patient survival for cobalt plaque versus enucleation in a larger group of patients for a longer follow-up time for equally sized, aged, and located tumours by the same methods of analysis as have previously been described. Ocular survival, visual acuity, and radiation complications from cobalt plaquing, all important considerations in terms of type of treatment, were not included in this study but will be examined at a later date.

In conclusion, as in prior studies, overall patient survival for cobalt plaque is better than enucleation. As in prior studies of enucleation, survival for cobalt plaque is poorest with anterior tumours and is best with smallest tumours.

For equal sized tumours, at the same location with equal follow-up, survival with cobalt plaque is at least equal to survival with enucleation. Therefore, if enucleation for a uveal melanoma is planned, cobalt plaque should be considered: the cure rate is the same, and obviously ocular survival, vision, and cosmesis are better.

\section{References}

1 Flocks M, Gerende J, Zimmerman LE. The size and shape of malignant melanomas of the choroid and ciliary body in relation to prognosis and histologic characteristics: a statistical study of 210 tumours. Ophthalmology 1955; 59: 740-58.

2 McLean IW, Foster WD, Zimmerman LE. Prognostic factors in small malignant melanomas of choroid and ciliary body. Arch Ophthalmol 1977; 95: 48-58.

3 Shammas HF, Blodi FC. Prognostic factors in choroidal and ciliary body melanomas. Arch Ophthalmol 1977; 95: 63-9.

4 Seddon JM, Albert DM. A prognostic factor study of diseasefree interval and survival following enucleation for uveal melanoma. Arch Ophthalmol 1983; 101: 1894-9.

5 Stallard HB. Radiotherapy for maligant melanoma of the choroid. Br J Ophthalmol 1966; 50: 147-55.

6 MacFaul PA. Local radiotherapy in the treatment of malignant melanoma of the choroid. Trans Ophthalmol Soc UK 1977; 97 : 421-7.

7 Ellsworth RM. Cobalt plaques for melanoma of the choroid. In: Jakobiec FA, ed. Ocular and adnexal tumours. Birmingham: Aescalapius, 1978: 76-9.

8 Migdal CS. Choroidal melanoma: the role of conservative therapy. Trans Ophthalmol Soc UK 1983; 103: 34-8.

9 Brady LW, Shields JA. Posterior uveal melanomas. In: Phillips TL, Postenmaa DA, eds. Radiation oncoloy annual. New York: Raven Press, 1984: 1.

10 Kaplan EL, Meier P. Nonparametric estimation from incomplete observations. J Am Stat Assoc 1958; 53: 457-81.

11 Cox DR. Regression models and lifetables. J R Stat Soc Ser B 1972; 34: 187-220.

12 Gass DM. Comparison of prognosis after enucleation vs. cobalt60 irradiation of melanomas. Arch Ophthalmol 1985; 103: 916-23.

Accepted for publication 18 May 1987. 